\title{
Giant-Magnetic-Resistance - An Aplication in Nondestructive Testing
}

Pries, Heiko

Volkswagen AG

Brieffach 1437/2

38436 Wolfsburg

\begin{abstract}
Nondestructive testing (NDT) of steel parts is of great interest for industrial manufacturers to assure their quality. In some cases all parts have to be tested before or after being processed or sold. Only NDT methods can provide maximum quality at low cost.

One of the most serious defects that might occur in production is surface flaws. These flaws need to be detected as soon as possible to allow real-time improvement within the production process. Automated NDT systems are suitable for online process monitoring as well as identifying process deviations, especially with regard to the statistical reproducibility of results.

A well-known NDT method for surface flaws in ferritic steel parts is magnetic particle inspection (MPI). The presence of a flaw leads to a perturbation of the magnetic flux in the investigated area, which causes a leakage of the magnetic field. The most common method of detecting this leakage utilizes finely divided particles, which are held in suspension by a suitable liquid. The particles are often colored or coated with fluorescent dyes, which emit under ultraviolet light (UV). The inspection is usually carried out manually by an employee, often within the assembly line production. Although some automated systems based on computer-aided image analysis are in use, it has yet to become a commonly practiced method.

Magnetoresistance (MR) sensors and scanning techniques are applicable in detecting small magnetic fields. In recent years some studies focused on first applying AMR sensors and later also GMR sensors in NDT techniques.

The paper will give a short review on magnetic particle inspection and will introduce a well suited GMRsensor made by SENSITEC that satisfies NDT requirements. An example is shown of how it may be used for constructing an automated in situ testing system for complex automotive parts, such as a steering rack. Results from laboratory evaluation on real part samples are given and discussed with regard to the sensor characteristics.
\end{abstract}

\section{Magnetic Particle Inspection}

Magnetic particle inspection (MPI) is a well-known NDT method for surface inspection of ferritic steel parts. An externally applied magnetic field is deformed by changing magnetic properties of the testing body. This deformation of the magnetic field is not limited to the immediate vicinity of the defect but extends for a considerable distance. The presence of a flaw leads to a perturbation of the magnetic flux, which causes a leakage of the magnetic field at the surface. For the following the flaw is considered to be a rectangular groove and the leakage is divided into its tangential and normal component as shown in Figure 1. 

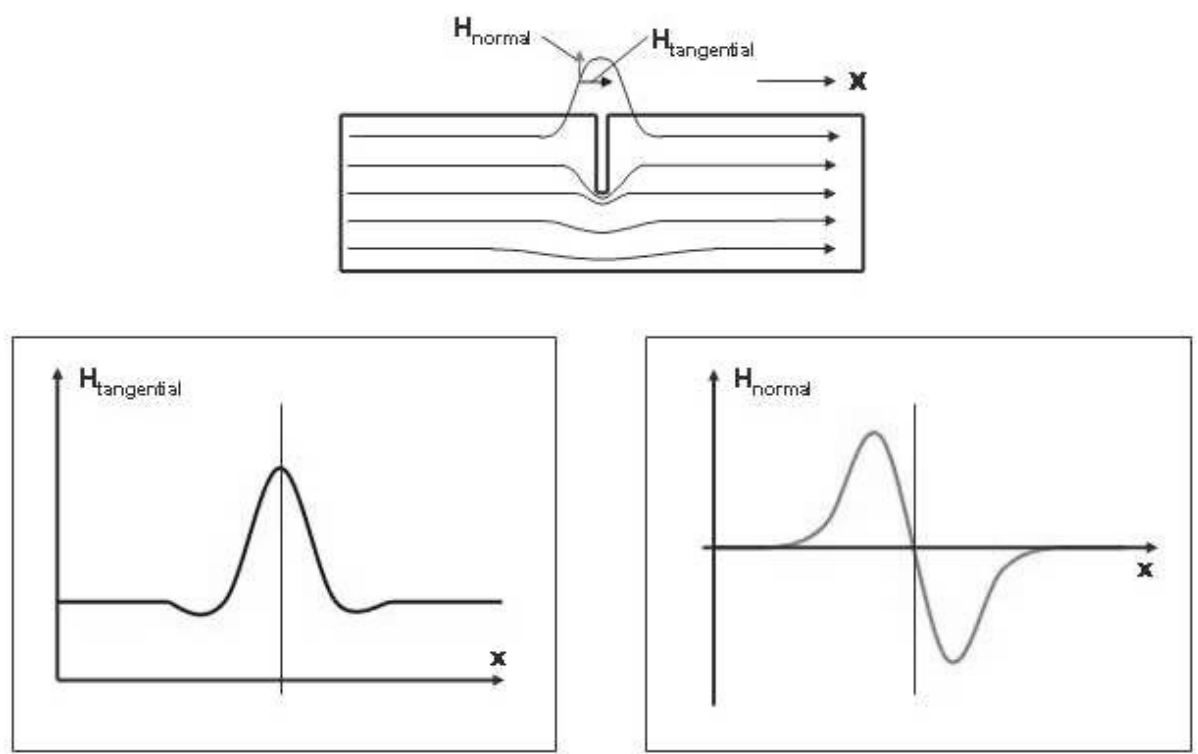

Figure 1: Tangential and normal component - schematic [1]

The most common method of detecting this leakage incorporates finely divided magnetic particles, which are held in suspension by a suitable liquid to improve mobility. Due to the interaction of magnetic forces the particles are attracted to the flaw and coalesce at the leakage. The particles are often colored or coated with fluorescent dyes, which emit under ultraviolet light (UV). The inspection is usually carried out manually by a worker, often within the assembly line production.

Fully automated MPI systems use computer-aided image analysis software and camera techniques. Systems are offered by different companies like Tiede, Karl Deutsch, WR Automation and KDFluxtechnic. Although some systems are in use, it has yet to become a commonly practiced method in automotive industry.
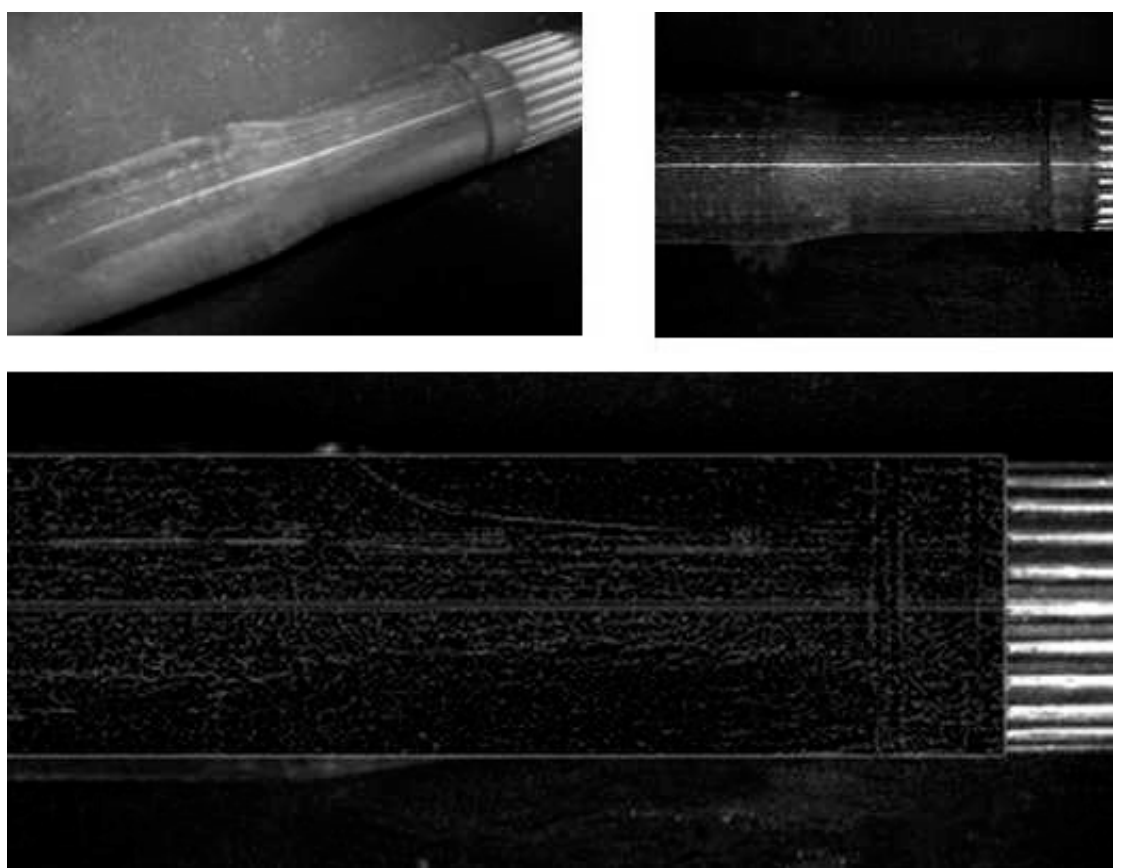

Figure 2: MPI result and Tiede OptoTec® image of a driveshaft 


\section{Sensor Based Magnetic Flux Leakage Detection (MFL)}

Magnetoresistance (MR) sensors and scanning techniques are applicable in detecting small magnetic fields. In recent years some studies focused on first applying AMR sensors and later also GMR sensors in NDT techniques like eddy current and magnetic flux leakage detection. Sensor-based MFL uses the interaction of the magnetic field with the probe to record an electric signal, which can be processed by data analysis tools.

Crack detection within an industrial environment requires specifically adapted probes and measurement techniques. With regard to all magnetic noises such as inhomogeneous excitation fields a self-compensating sensor is the most advantageous. High spatial resolution and high sensitivity is needed for further mathematical operations to provide a maximum of information about the detected flaw. Scanning a complex test body (i.e. steering rack) within cycle time can only be realized using an appropriately shaped array of sensors which have to be of miniaturized size. A sensor complying with these conditions is produced by Sensitec $\AA$ and is shown in Figure 3below.
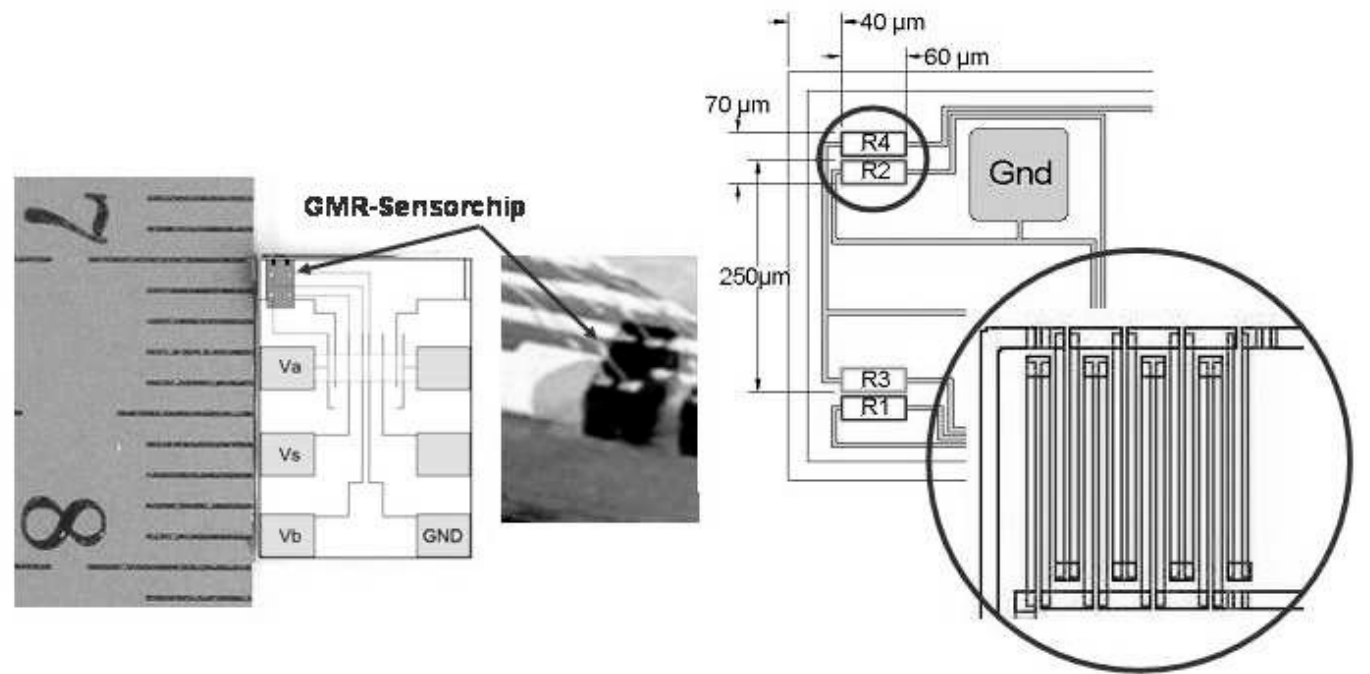

Figure 3: Detail of the Prototype Sensor used for application research [2]

The exchange bias direction of the sensor matches the normal component of the magnetic leakage due to the presence of a flaw. 


\section{Application Research}

The schematic setup of the probe scanning direction and the characteristic curve is given in Figure 4.

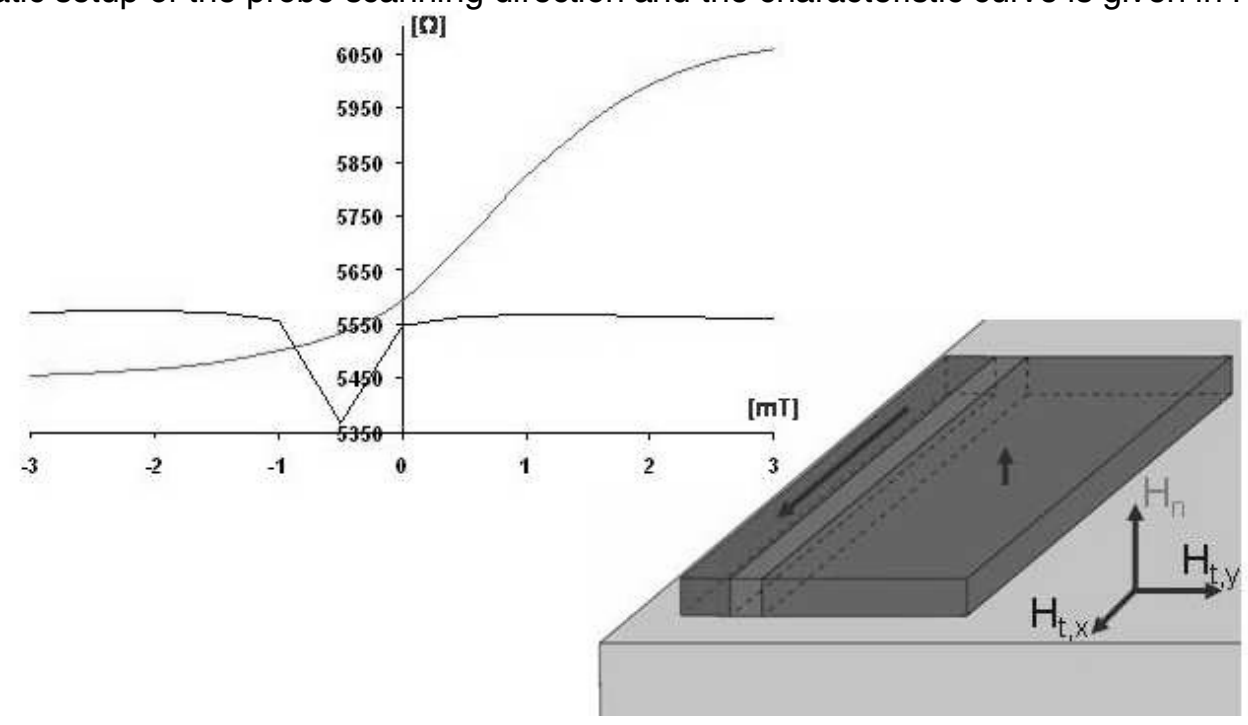

Figure 4: Setup of Sensor and testing body

The testing body is a welded part with several hot cracks. It was grounded to eliminate any disturbances due to scratches and surface textures. The signal of a scan orthogonal to the crack is provided in Figure 5.

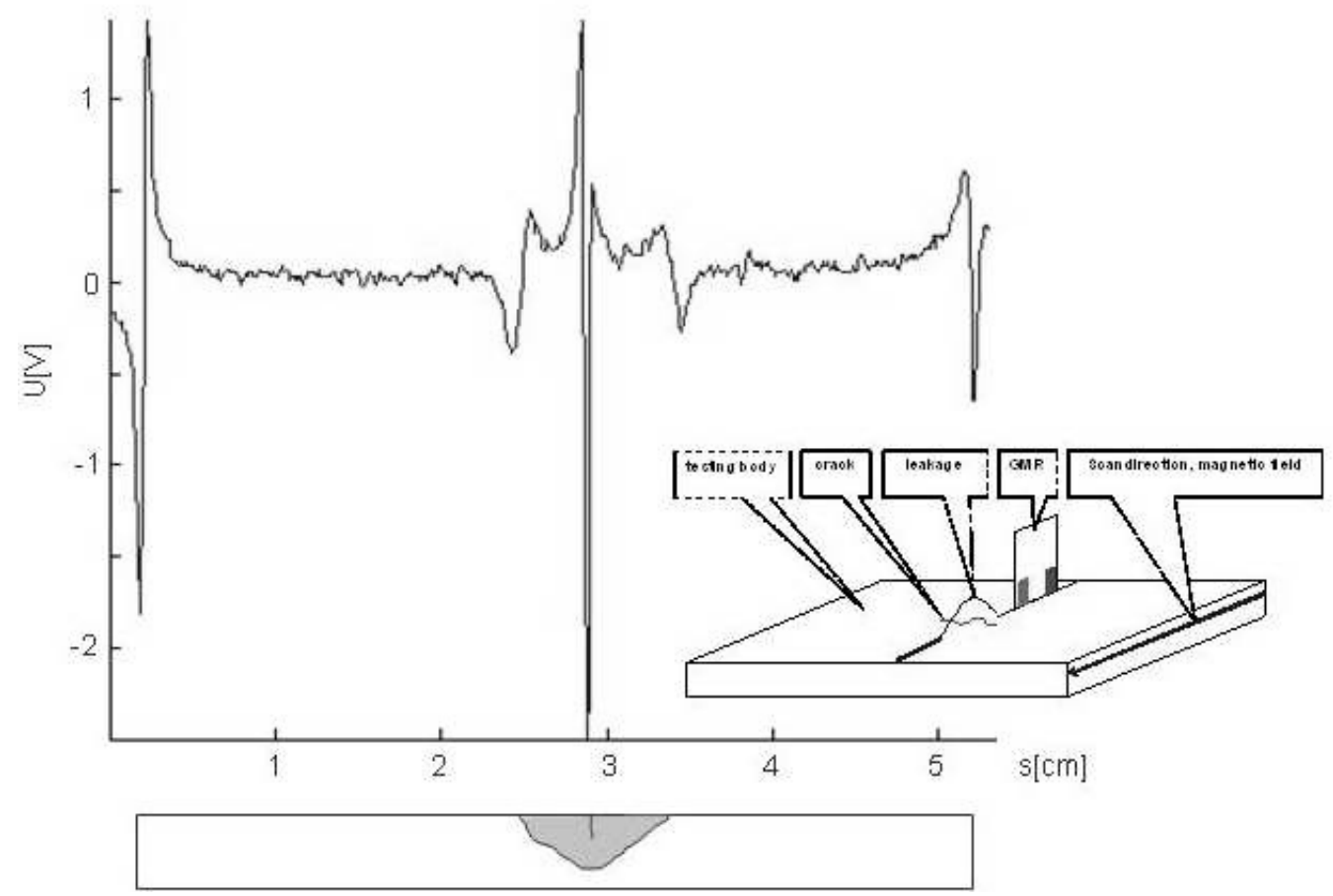

Figure 5: signal of a scan orthogonal to the crack

Scanning at high resolution with this setup provides a magnetic image of the magnetic field gradient above the surface which is compared to the UV-picture of the MPI below. 


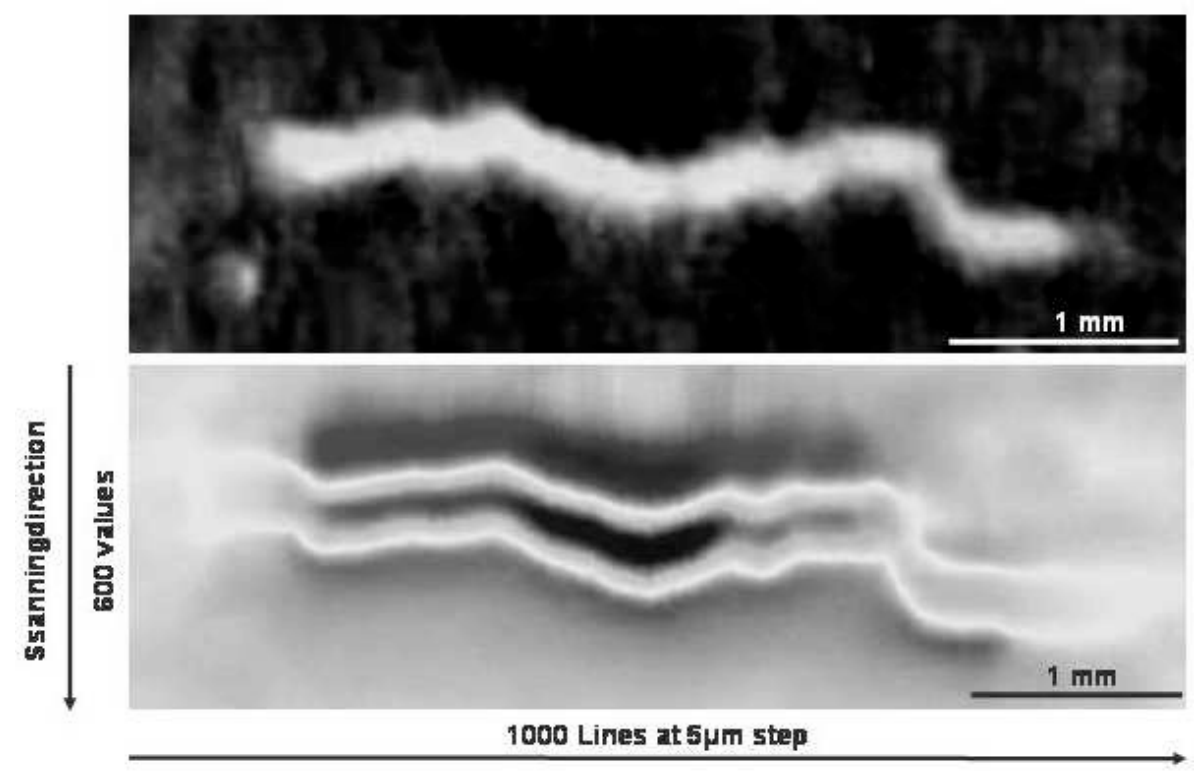

Figure 6: MPI and high resolution GMR-scan image

The main challenge for industrial use is to keep the distance between sensor and testing body minimized. The signal to noise ratio $(\mathrm{S} / \mathrm{N})$ declines with the distance as demonstrated in figure 7 .

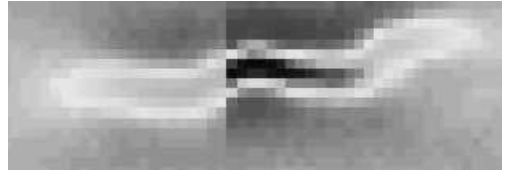

$\Delta=0 \mu \mathrm{m}$ $\mathrm{S} / \mathrm{N}=100 \%$

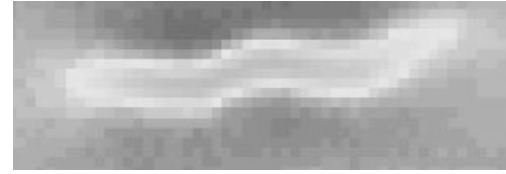

$\Delta=50 \mu \mathrm{m}$

$\mathrm{S} / \mathrm{N}=50 \%$

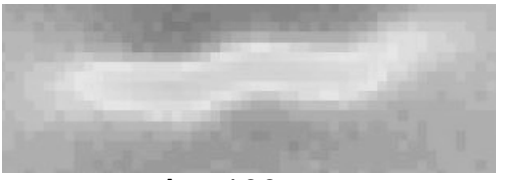

$\Delta=100 \mu \mathrm{m}$

$\mathrm{S} / \mathrm{N}=25 \%$

Figure 7: GMR-scan image with various distances

\section{References}

[1] DIN EN 54136:1988

[2] Sensitec, Unterlagen zum Prototypensensorwaver 101280 